\title{
RADIOCIRURGIA ESTEREOTÁXICA NO TRATAMENTO DE METÁSTASES CEREBRAIS: UMA REVISÃO NARRATIVA
}

STEREOTAXIC RADIOSURGERY IN THE TREATMENT OF CEREBRAL METASTASIS: A NARRATIVE REVIEW

\section{Kátia Rayanne Rodrigues Moreno}

Discente do Curso Superior de Tecnólogo em Radiologia, Faculdade Evangélica de Ceres-GO E-mail: katia_rayanne@ @otmail.com

\section{Carla Danielle Dias Costa}

Docente da Faculdade Evangélica de Ceres.

E-mail: carladaniellebm@gmail.com

\section{RESUMO}

INTRODUÇÃO: A Radiocirurgia Estereotáxica (RCE) é uma modalidade de radioterapia, minimamente invasiva e indolor, que utiliza altas doses de radiação em uma única seção para tratamento de lesões intracranianas extereotaxicamente definidas. Por apresentar resultados satisfatórios ou superiores aos obtidos por intervenção cirúrgica ou outros tratamentos, é indicada para o tratamento de patologias como as metástases cerebrais. OBJETIVO: Compreender as aplicações da radiocirurgia no tratamento das metástases cerebrais, apontando as vantagens e desvantagens da sua utilização, enfatizando a efetividade da técnica para este tipo de tratamento. METODOLOGIA: Foi realizada uma revisão narrativa descritiva utilizando os bancos de dados SCIELO, BVS, e LILACS. Obteve-se uma população de 9.687 arquivos em português, sem delimitação temporal. E amostra de 38 artigos que se mostraram pertinentes ao tema. RESULTADOS E DISCUSSÕES: Desde a década de 80 a RCE vem obtendo sucesso, com pouca agressividade ao paciente, no tratamento de lesões metastáticas cerebrais. CONCLUSÃO: A radiocirurgia estereotáxica demonstra ser uma técnica eficiente no tratamento das metástases cerebrais. Tendo como maior vantagem a administração máxima da dose prescrita somente no volume alvo, protegendo assim as regiões ao redor. Porém a técnica possui algumas limitações como baixa aplicabilidade em lesões metastáticas maiores que $3 \mathrm{~cm}$, e o fato das respostas ao tratamento não serem imediatas.

Palavras chave: Radiocirurgia. Estereotaxia. Radioterapia. Cérebro. 


\begin{abstract}
INTRODUCTION: Stereotaxic radiosurgery (RCE) is a minimally invasive and painless radiotherapy modality that uses high doses of radiation in a single section to treat exteroeotaxically defined intracranial lesions. Does it presents satisfactory results or superior to those obtained by surgical intervention or other treatments, it is indicated for the treatment of pathologies such as cerebral metastasis. OBJECTIVE: Understand the applications of radiosurgery in the treatment of cerebral metastasis, pointing out the advantages and disadvantages of its use, emphasizing the effectiveness of the technique for this type of treatment. METHODS: A descriptive narrative review was performed using the SCIELO, BVS, and LILACS databases. We obtained a population of 9,687 files in Portuguese, with out temporal delimitation. And a sample of 38 articles that were relevant to the theme. RESULTS AND DISCUSSIONS: Since the 1980s, RCE hás been successful, with little aggression to the patient, in the treatment of cerebral metastatic lesions. CONCLUSION: Stereotaxic radiosurgery hás been shown to be an efficient technique for the treatment of cerebral metastasis. Having the greatest advantage is the maximum administration of the prescribed dose only in the target volume, thus protecting the surrounding regions. However, the technique has some limitations such as low applicability in metastatic lesions greater than $3 \mathrm{~cm}$, and the fact that the responses to treatment are not immediate.
\end{abstract}

Keywords: Radiosurgery. Stereotaxic. Radiotherapy. Cerebral.

Endereço para correspondência: Av. Brasil, s/n, Qd 13, Morada Verde, Ceres-Goiás, Brasil. CEP: 76300-000. Fone: (62) 3323-1040.

\title{
INTRODUÇÃO
}

O câncer é uma doença que caracteriza-se pelo crescimento descontrolado de células do organismo causado por mutações genéticas (alterações no DNA das células) (BRASIL, 2011). Os fatores que provocam essas mutações podem surgir sem nenhum estímulo específico ou por exposição a agentes carcinogênicos como fatores genéticos, químicos, físicos, biológicos, e estilo de vida. (BRASIL, 2008). As células que apresentam crescimento desordenado, são consideradas células cancerosas e devido sua patogenicidade (capacidade do agente invasor em causar doenças) são capazes de se moverem para outras regiões do corpo, dando origem a tumores em diversos locais do organismo. A esses novos focos, dá-se o nome de metástases (BRASIL, 2011).

Uma estimativa realizada para o Brasil no período de 2016-2017 indicou a ocorrência de aproximadamente 600 mil novos casos de cânceres, destes, os mais incidentes são os 
cânceres de pele não melanoma, próstata, mama, pulmão, intestino, estômago, cavidade oral, e colo do útero. É previsto que em 2025 o câncer atingirá $80 \%$ da população mundial, totalizando 20 milhões de novos casos (BRASIL, 2015).

Os tratamentos do câncer objetivam a cura, o prolongamento da vida e a melhora da qualidade de vida dos pacientes. E podem ser feitos através de três principais formas: cirurgia, quimioterapia e radioterapia (BRASIL, 2011). A cirurgia trata-se da remoção total ou parcial do tumor, promovendo ao paciente um efeito curativo ou paliativo (BRASIL, 2008). A quimioterapia é um tipo de tratamento a base de medicamentos quimioterápicos (BRASIL, 2011). E a radioterapia que tem como característica a utilização de radiação ionizante sob a área em que está localizado o tumor, com o objetivo de suprimir as células tumorais do órgão e criar uma margem de segurança incluindo os tecidos adjacentes ao tumor a fim de eliminar quaisquer vestígios das células cancerosas (LIMA et al., 2014).

A radioterapia pode ser dividida em três tipos de procedimentos: Teleterapia, Braquiterapia, e a Radiocirurgia. A Teleterapia fundamenta-se no tratamento à distância entre o equipamento de radioterapia que emitirá radiação e a região que será tratada, ou seja,a fonte de radiação é externa ao paciente. Na Braquiterapia, a fonte de radiação fica em contato com os tecidos que serão irradiados, inclusive inseridos dentro do órgão a ser tratado. E por último, a Radiocirurgia, na qual se administra em uma única ocasião, uma alta dose de radiação $\chi$ (em procedimentos nos quais os aceleradores lineares são empregados) ou radiação $\gamma$ (nos casos em que se utiliza o aparelho Gamma Knife) (LIMA et al., 2014). Essas doses são administradas em um alvo (região onde se localiza o tumor) estereotaxicamente definido (LUNDGREN et al., 2012). O termo estereotáxico refere-se ao mapeamento em três dimensões utilizado como orientação no procedimento radiocirúrgico (TEIXEIRA, 2015).

A Radiocirurgia Estereotáxica (RCE) é um procedimento realizado em hospitais especializados, no entanto não exige internação do paciente. Geralmente, a aplicação é feita em dose única, mas pode ser repetida quando necessário para tratar doenças recorrentes (GOMES et al., 2014). É uma técnica que na maioria das vezes apresenta resultados similares ou até mesmo superiores aos resultados obtidos em tratamentos cirúrgicos. É minimamente invasiva, indolor, não sendo necessária aplicação de anestesia geral. Entretanto se faz necessário o uso de anestesia local na técnica frame, pois a imobilização do órgão é feita a partir da fixação de um arco craniano, já na técnica frameless não se faz necessário o uso da mesma, sendo que a imobilização é feita utilizando uma máscara termoplástica de maneira não invasiva (ALMEIDA et al., 2016). 
A RCE é indicada para o tratamento de diversos tipos de tumores com até $3 \mathrm{~cm}$ de extensão, como também é utilizada no tratamento de metástases cerebrais, más formações vasculares, dores resistentes à medicação, principalmente a dor de origem oncológica e certas neuralgias (dores intensas ao longo dos nervos do corpo humano), e demais patologias (GOMES et al., 2014). Muitos artigos vêm sendo publicados reforçando as vantagens da RCE em metástases cerebrais (GOMES et al., 2014; MALDAUN, 2006; CANTERAS, 2005; SANTOS et al., 2001; FARIA et al., 1995).

Embora a RCE venha sendo utilizada desde 1951 no mundo, poucas informações são disponibilizadas à população sobre esta técnica de tratamento em metástases cerebrais, sendo assim, se faz necessário um levantamento sobre a mesma, a fim de se conhecer a empregabilidade dela frente às outras metodologias de tratamento.

O objetivo deste artigo foi compreender as aplicações da Radiocirurgia no tratamento de metástases cerebrais, apontando quais as vantagens e desvantagens mais recorrentes ao emprego deste método a fim de se enfatizar a efetividade da técnica.

\section{METODOLOGIA}

O presente estudo refere-se a uma revisão narrativa descritiva acerca do tratamento de metástases cerebrais utilizando como técnica a Radiocirurgia. Os bancos de dados: Scientific Eletronic Library OnLine (SCIELO), Biblioteca Virtual de Saúde (BVS) e a Literatura Latino-Americana e do Caribe em Ciências da Saúde (LILACS) foram consultados para a busca de informações no período de Agosto a Novembro de 2017. As palavras-chaves utilizadas foram: radiocirurgia, estereotaxia, radioterapia e cérebro. As mesmas foram pesquisadas individualmente e/ou associadas por meio de indicadores booleanos (and, or, not).

Como critérios de inclusão foram utilizados artigos completos e originais, em português, sem restrição temporal. Quanto ao tipo de artigos foram selecionadas revisões bibliográficas, assim como trabalhos com delineamento experimental (ensaios clínicos, randomizados) ou observacional (estudos de caso-controle, estudos de coorte) que abordavam sobre a Radiocirurgia em tratamento de metástases cerebrais.

A população do estudo foi composta por toda a literatura disponível $(9.687$ em português) nos bancos de dados SCIELO, BVS e LILACS. Quanto à amostra, 38 artigos pertinentes foram selecionados. 
Para a análise de dados, realizou-se a leitura de todo material, recolhendo as principais informações referentes aos objetivos propostos pela pesquisa, para posteriormente estabelecer a compreensão e ampliação do conhecido sobre o tema pesquisado.

\section{RESULTADOS E DISCUSSÃO}

Metástase é o processo caracterizado pelo surgimento de uma lesão tumoral em novas estruturas, a partir de uma lesão tumoral primária. Esta situação ocorre devido a uma série de possibilidades como: a separação e o deslocamento de células tumorais primárias, invasão nas vias linfáticas ou sanguíneas, sobrevivência dessas células na circulação, união à camada externa da estrutura em que irá se instalar, e a reprodução no órgão invadido (FILHO, 2013). Para que ocorra a metastatização de um tumor e o mesmo se tornar radiologicamente visível, o ambiente deve ser propício para receber as células tumorais e suportar seu crescimento (FERNANDEZ, 2012).

Qualquer tipo de câncer pode metastatizar para o sistema nervoso central, os mais preponderantes são os cânceres pulmonares, mamários, melanomas, colorretais e renais (MALDAUN, 2006). Aproximadamente 20 a 40\% dos pacientes com câncer desenvolvem metástases cerebrais (FERNANDEZ, 2012).

As metástases cerebrais são lesões cerebrais adequadas para o tratamento radiocirúrgico devido sua forma esférica (FARIA et al., 1995), por geralmente serem menores que $3 \mathrm{~cm}$ (BRANDT et al., 1995) e por algumas estarem situados em áreas inacessíveis cirurgicamente como o tronco cerebral (que se situa entre a medula espinhal e o diencéfalo) e área motora (que é responsável pelos movimentos realizados pelo organismo) (MASCARENHAS et al., 2004).

\section{Radiocirurgia Estereotáxica (RCE)}

O conceito de Radiocirurgia foi apresentado em 1951 por Lars Leksell (CANTERAS, 2005) que devido à letalidade da cirurgia neurológica na época ser muito elevada, buscava uma alternativa para aumentar a qualidade e o tempo de vida dos pacientes (LUNDGREN, 2011), além de tentar estabelecer um método não invasivo para lesões intracranianas de difícil acesso, praticamente inoperáveis (TEIXEIRA, 2015).

Em 1951 em Estolcomo, Leksell tratava com RCE seu primeiro caso, uma neuralgia do trigêmeo, utilizando raios-x como fonte de radiação e sistema estereotáxico criado por ele dois anos antes. O tratamento foi um sucesso, obtendo melhora da dor (SANTOS, 2003; 
LUNDGREN, 2011). Inicialmente sua utilização era para tratar doenças funcionais cerebrais de localizações específicas, principalmente o transtorno obsessivo compulsivo. Entre 1984 e 1985, a RCE começou a ser utilizada no tratamento de metástases cerebrais por um grupo de pesquisadores do departamento de neurocirurgia da Universidade de Heidelberg, e mostrou redução do volume e redução do edema nas regiões ao redor da lesão (MALDAUN, 2006; STURM et al., 1987). Esses efeitos contribuem para uma melhora do quadro clínico e consequentemente um período maior de sobrevida e melhor qualidade de vida aos pacientes que apresentam metástases cerebrais (WELTMAN; BRANDT, 2004).

A RCE não é um procedimento cirúrgico, portanto não é necessária a abertura do crânio para a realização da técnica (BARBOSA, 2010; LOPES et al., 2004). Geralmente o procedimento consiste na administração de radiação em um único momento. Este método reduz o tempo total do tratamento, além de diminuir custos não médicos como o deslocamento do paciente e dos acompanhantes, hospedagem, alimentação e retorno às atividades do cotidiano (MASCARENHAS et al., 2004). A RCE também pode ser associada a outras técnicas de tratamento (CANTERAS, 2005), como por exemplo a radioterapia de cérebro total, a microcirurgia, ou ambas (GOMES et al., 2014), assim como complemento a cirurgia convencional (BEHARA; FERANCIC; SCAFF 1987).

Este procedimento é indolor, em sua maioria não gera sequelas pós-cirúrgicas, além de ter como característica a capacidade de diminuir os riscos de infecções (BARBOSA, 2010). Portanto, a RCE vem sendo realizada para o tratamento de diversas patologias intracranianas (GOMES et al., 2014) e até mesmo extracranianas (JOAQUIM et al., 2013).

Nos tratamentos com RCE a participação de uma equipe multidisciplinar é fundamental para extrair o melhor rendimento possível do tratamento. Esta equipe geralmente é constituída por neurocirurgiões, neurorradiologistas, radioterapeutas, técnicos/tecnólogos em radioterapia, físicos, além de requerer uma estrutura paramédica de enfermeiros e outros profissionais especializados (ESCOLASTICO, 2011).

\section{Equipamentos utilizados na radiocirurgia}

Desde a década de 80 pacientes com metástases cerebrais vem sendo tratados com RCE, através de um equipamento chamado Gamma Knife, assim como através dos aceleradores lineares (LINACs). Ambos são sistemas usados na radiocirurgia e oferecem segurança e eficácia ao tratamento (GOMES et al., 2014). Aparelhos como o CyberKnife, tem como característica ser um acelerador linear que ao invés da estereotaxia, utiliza imagens em 
tempo real para localizar o alvo (ESCOLASTICO, 2011), podendo assim ser utilizado para tratar lesões extracranianas, como coluna vertebral (PANULLO et al., 2011). Partículas pesadas aceleradas em cíclotrons, também são utilizadas como sistemas que possibilitam a melhor distribuição da dose, sendo mais adequado para tratar lesões amplas ou adjacentes a estruturas vitais (SOUZA, 2010), porém devido o seu alto custo, o uso deste aparelho é limitado (CASTRO et al, 2006).

O sistema Gamma Knife foi o primeiro dedicado exclusivamente a RCE (BARBOSA, 2010). Foi desenvolvido em 1967 (LUNDGREN, 2011) para tratar lesões no compartimento intracraniano (SOUZA, 2010). É um aparelho que possui 201 fontes de Cobalto 60 que geram múltiplos feixes de radiação $\gamma$ (JUSTINO et al., 2004; ESCOLASTICO, 2011). Já os sistemas LINACs utilizam feixes de raio-X para atingir os alvos pré-determinados, foram empregados em 1974 (ESCOLASTICO, 2011) e são os mais utilizados no Brasil e no mundo (CASTRO et al., 2006) por oferecem menor custo comparado ao sistema Gamma Knife (TEIXEIRA, 2015), devido a isso e a sua utilidade inclusive na radioterapia convencional atuando assim em outras regiões do corpo (CASTRO et al., 2006). O Brasil possui 259 centros de radioterapia, desses aproximadamente $14 \%$ oferecem a RCE como tratamento, entre estes, somente dois centros, localizados em São Paulo e Paraná, executam esta técnica com o Gamma Knife, e os demais utilizam aceleradores lineares (TEIXEIRA, 2015).

\section{Tratamento de metástases cerebrais por meio de radiocirurgia}

A realização da avaliação do Karnofsky Perfomance Status - KPS (medida que avalia as condições neurológicas) indica o tratamento, no caso a RCE, se fixa o equipamento estereotáxico (frame ou frameless) (BEHARA; FERANCIC; SCAFF, 1987) para localizar precisamente os alvos a serem tratados (BARBOSA, 2010; SOUZA, 2010), realiza-se exames de Tomografia Computadorizada (TC) e Ressonância Magnética (RM) para obtenção de imagens, delineamento do volume-alvo e reconstrução volumétrica (em três dimensões) (SOUZA; MONTI, 2001).

Os dois métodos para obtenção de imagens devem ser usados, pois a TC oferece maior precisão espacial, enquanto a RM melhor sensibilidade anatômica (SOUZA, 2010). Fundemse as imagens de TC e RM (Figura 1) para obter maiores informações para o planejamento da irradiação da lesão (LUNDGREN, 2011). 


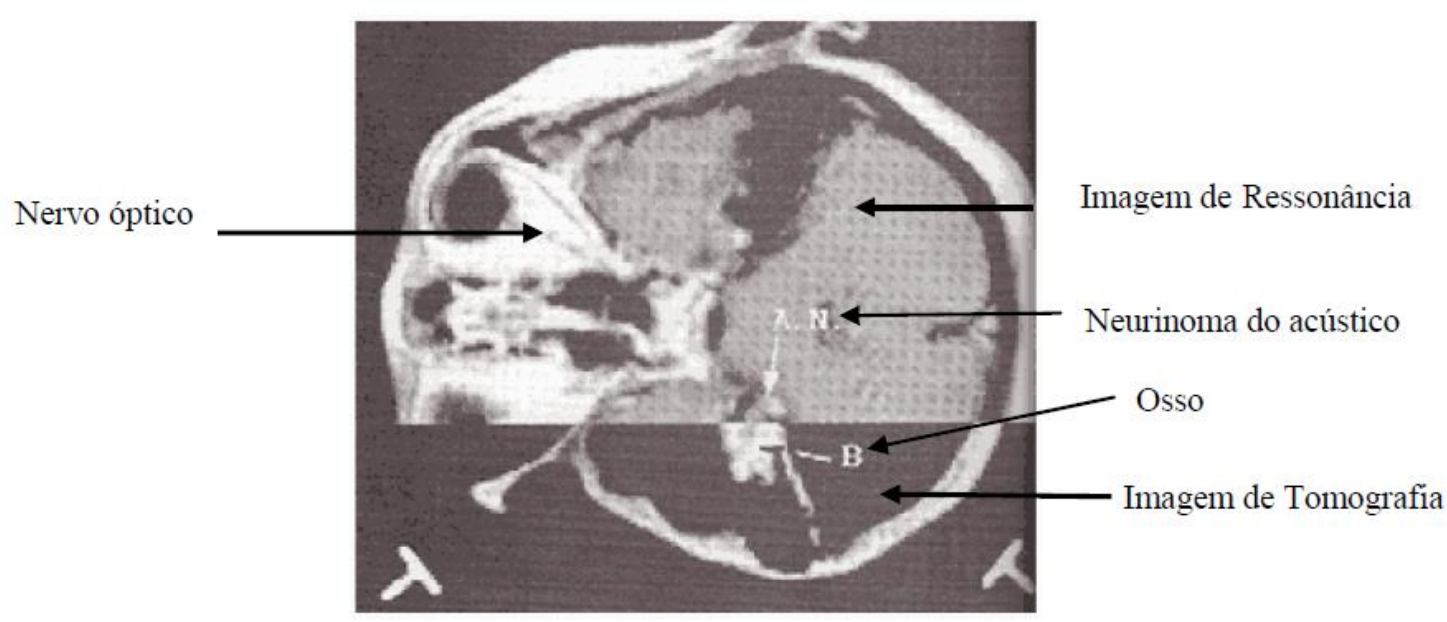

Figura 1- Imagem do crânio com a fusão de imagem de tomografia e ressonância (KOOY et al., 1994)

Realizada a fusão das imagens, há a transferência destas para um sistema de planejamento (LUNDGREN, 2011) no qual se faz a delimitação do(s) volume(s) alvo(s) e estruturas a se proteger, seguido por definição da dose de irradiação que será usada, posicionamento do paciente e avaliação deste posicionamento, o qual deve ser realizado exatamente como na aquisição das imagens, para garantir equivalência máxima na localização da lesão que será irradiada (ESCOLASTICO, 2011). Posteriormente, são realizados controles de qualidade (DELGADO et al., 2006), depois é aplicado o tratamento, e ulteriormente o aparelho estereotáxico é retirado (BEHARA; FERANCIC; SCAFF 1987). Podendo assim, o paciente voltar as suas atividades normais no dia seguinte (SOUZA, 2010).

A intenção do tratamento ideal é liberar $100 \%$ da dose prescrita no volume-alvo e nenhuma dose no tecido normal, porém na prática, isto não é possível. Então o principal objetivo do plano de tratamento é conformar a dose prescrita no volume-alvo tão próximo quanto possível, e exercer a menor dose possível nas estruturas críticas do cérebro, fora do volume-alvo (JUSTINO et al., 2004).

Um estudo realizado no período de 1988 a 1993 avaliou 52 pacientes com metástases cerebrais tratados com RCE. Destes, 25 melhoraram o quadro de metástases, 20 prosseguiram inalterados, três pioraram e quatro não se obtiveram informações (FARIA et al., 1995). No estudo de Brandt et al. (1995) foram analisados 12 pacientes, destes oito tiveram melhoria, dois se mantiveram inalterados, um piorou após período inicial de estabilização devido o avanço das metástases, e um veio a óbito em decorrência da sua doença primária. Já no trabalho de Fernandez (2012) foram acompanhados 39 pacientes, dos quais somente quatro fizeram o tratamento através da RCE. Dos 35 indivíduos que não realizaram a RCE, 25 destes 
morreram. Em relação aos quatro pacientes que foram submetidos a RCE, dois tiveram aumento de nove meses em sua sobrevida e os outros dois vieram a óbito. Com esses estudos, foi possível observar a eficácia e pouca agressividade da radiocirurgia estereotáxica.

\section{Doses de Radiação utilizadas nos tratamentos de Metástases Cerebrais}

As doses de radiação administradas no tratamento de metástases cerebrais por RCE variam de acordo com o tamanho da lesão (FERNANDEZ, 2012). Quanto maior a lesão, menor deverá ser a dose administrada para prevenir complicações (AMARAL, 2010). As doses seguras estabelecidas pelo Radiation Therapy Oncology Group variam de 15 a 24 Gy, dependendo do volume da lesão (LUNDGREN, 2011).

Fernandes (2012) e Maldaun (2006) afirmam que doses de 24Gy devem ser aplicadas somente em lesões inferiores a 2cm. 18Gy em lesões entre 2,1 a $3 \mathrm{~cm}$. A acurácia da dosagem é de grande importância, pois qualquer desvio da dose prescrita é considerado mais crítico que em outras modalidades de tratamento radioterápico, já que são utilizadas altas doses de radiação (TEIXEIRA, 2015). A prescrição da dose é baseada na tolerância das estruturas metastáticas e adjacentes. As vias ópticas são as mais sensíveis a exposição à radiação e não devem receber uma dose maior do que $8 \mathrm{~Gy}$ para evitar complicações permanentes (CASTRO et al., 2006).

\section{Vantagens e desvantagens da radiocirurgia no tratamento de metástases cerebrais}

Quanto as vantagens da RCE valem ressaltar que há um controle no crescimento dos tumores metastáticos de aproximadamente $80 \%$ nos pacientes tratados com RCE, e a diminuição dos sintomas (como cefaléia e estado mental alterado) ocorre oferecendo ao paciente oncológico maior qualidade de vida (FERNANDEZ, 2012).

Objetivando amenizar esses sintomas e melhorar a qualidade de vida e, portanto, aumentar sua sobrevida, os pacientes submetidos à RCE devem preferivelmente possuir um KPS maior que 70 (PANULLO et al., 2011), tornando inviável a utilização para aqueles que possuam KPS baixo, devido o quadro clinico do paciente (Quadro 1) (WELTMAN; BRANDT, 2006).

\begin{tabular}{|ll|}
\hline ESCALA & PERFORMANCE STATUS \\
\hline 100 & Normal, sem queixas e sem evidências da doença \\
90 & Capacidade para atividades normais, sintomas leves \\
80 & Atividade normal com esforço, sintomas moderados \\
70 & Incapacidade para atividades normais \\
60 & Independente na maioria das atividades pessoais, requer assistência ocasional \\
\hline
\end{tabular}




\begin{tabular}{|ll|}
\hline 50 & Requer considerável assistência e cuidados frequentes \\
40 & Dependente, incapacitado, requer assistência e cuidados especiais \\
30 & Incapacidade severa, hospitalizado, sem risco de morte iminente \\
20 & Muito doente, requer suporte clínico ativo \\
10 & Moribundo, óbito iminente ou próximo \\
0 & Óbito \\
\hline
\end{tabular}

Quadro 1- Karnofsky Performance Status (WELTMAN; BRANDT, 2006).

Atualmente no Brasil, além da RCE estar disponível na rede privada, também se encontra na rede pública, através do Sistema Único de Saúde (SUS) do governo federal (MAIA, 2015).

Em relação à cirurgia, a RCE possui maior vantagem por não necessitar da abertura do crânio e, portanto, diminuir o risco de infecções (BABOSA, 2010), e por conseguir acesso em áreas cirurgicamente inviáveis. Em comparação com a radioterapia, a RCE também possui maior vantagem, por ser realizada em menos seções reduz o tempo de tratamento, os custos (MASCARENHAS et al., 2004), e poupa os tecidos vizinhos sadios à lesão do paciente, de irradiação ao administrar uma dose focal de radiação no alvo (SANTOS et al., 2001).

É primordial o controle da doença metastática para a prevenção e atenuação dos sintomas neurológicos que debilitam os pacientes, visando uma sobrevida longa e com qualidade (GOMES et al., 2014). A sobrevida mediana destes pacientes sem qualquer tratamento é de apenas sete semanas, com morte normalmente causada pelo aumento da pressão intracraniana (SANTOS et al., 2001). Com a aplicação da técnica da RCE observouse um aumento na sobrevida de 10 meses em pacientes com uma ou duas metástases e de 6,5 meses naqueles com três ou mais lesões (ALEXANDER III et al., 1995). Assim como notado nos trabalhos de Metha et al. (1992), Kondziolka et al. (1999) e Weltman e Brandt (2006).

Assim como outros tratamentos, a RCE possui algumas limitações como a incapacidade de minimizar rapidamente os sintomas tumorais relacionados ao efeito de massa (compressão exercida pelo aumento da pressão intracraniana, empurrando estruturas cerebrais). A técnica também possui baixa aplicabilidade em lesões metastáticas maiores que $3 \mathrm{~cm}$ e a lesões localizadas a menos de $3 \mathrm{~mm}$ de estruturas radiossensíveis (estruturas com baixa tolerância a radiação) como nervos ópticos e quiasma (cruzamento destes nervos); e ocorrência de complicações como a radionecrose cerebral (morte de células cerebrais devido à radiação) que ocorre normalmente após três meses (AMARAL, 2010) em até 10\% dos pacientes tratados com RCE (GOMES et al., 2014). 
O tamanho do volume da lesão metastática a ser tratado é um fator importante, quanto maior a dimensão do mesmo, maiores são as chances de necrose e de repetição do tratamento (MALDAUN, 2006). E apesar das altas doses de radiação serem aplicadas somente no alvo, com o mínimo de radiação para os órgãos vizinhos, outras estruturas do corpo do paciente podem receber radiação secundária devido a disseminação por partes do aparelho, paredes da sala, vazamentos de radiação, e do próprio paciente (LUNDGREN; KHOURY; AZEVEDO, 2012). Portanto, a necessidade de controle de qualidade rigoroso em todos os procedimentos e cuidados singulares para garantir a eficácia de todos os sistemas envolvidos, sejam eles mecânicos, de radiação, ou planejamentos, se fazem necessários para garantir maior segurança do paciente (BRANDT et al., 1995).

Alguns erros como o planejamento incorreto, assim como a má execução de condutas planejadas, podem ocorrer durante o procedimento radiocirúrgico, e gerar uma consequência muito séria ou até mesmo fatal devido às altas doses de radiação que tem seu uso irreversível. Em estudo realizado por Teixeira (2015), ocorreram 227 acidentes em RCE entre 2004 a 2009 tendo como consequência dois óbitos e duas complicações com porvindouro óbito. Estes óbitos foram decorrentes de falhas de comunicação entre o físico médico e o técnico, e a falhas na interface dos softwares.

Para que erros como esses sejam evitados, melhorando os resultados do método e garantindo maior segurança ao paciente, aplica-se um rigoroso controle de qualidade em todo o procedimento (BARBOSA, 2010). Dentro deste controle tem-se a dosimetria como uma técnica de extrema importância para avaliar as doses de radiação recebidas pelo paciente, evitando danos em estruturas adjacentes e radiossensíveis. Portanto, deve-se sempre dar atenção ao controle de qualidade e a realização adequada da dosimetria a fim de se proteger o paciente (LUNDGREN; KHOURY; AZEVEDO, 2012).

\section{CONCLUSÃO}

A radiocirurgia estereotáxica é uma técnica eficaz e segura, que tem como principal vantagem a agressão mínima das estruturas adjacentes às que são tratadas, além de ser um procedimento normalmente realizado em seção única. Entretanto, necessita-se de alguns meses para obter melhora dos sintomas causados pelas lesões metastáticas.

O tratamento de metástases cerebrais com a RCE é eficaz e é comprovado por diversos autores. Diante de todos os benefícios apresentados pela mesma, vê-se a necessidade de ampliar as discussões tanto no âmbito médico, acadêmico assim como popular, a fim de 
torna-lá um tratamento acessível à população, levando a diminuição de gastos e melhoras na qualidade de vida dos pacientes que apresentam metástases cerebrais.

\section{REFERÊNCIAS}

ALEXANDER III, E.; MORIATY, T. M.; DAVIS, R. B.; WEN, P. Y.; FINE, H. A.; BLACK, P. M.; KOOY, H. M.; LOEFFLER, J. S. Stereotactic radiosurgery for the definitive, non invasive treatment of brain metástases. Journal of the National Cancer Institute, Oxford, v. 87, n. 1, p. 34-40,1995.

ALMEIDA, T. V. R.; JUNIOR, A. L. C.; PIEDADE, P. A.; SILVA, C. M.; MARINS, P.; ALMEIDA, C. M.; BRINCAS, G. R.B.; SOBOLI, D. S. Análise dos erros de posicionamento translacionais em radiocirurgia craniana frame e frameless com uso de objeto simulador antropomórfico. Revista Radiologia Brasileira. São Paulo, v.49, n. 2, p. 98-103, 2016.

AMARAL, F. Conhecimento atual sobre complicações maiores após irradiação cerebral total ou radiocirurgia. Acta Médica Portuguesa, Lisboa, v.23, n.1, p. 85-94, 2010.

BARBOSA, Nilséia Aparecida. Desenvolvimento de um simulador de cabeça para a aplicação no controle de qualidade de radiocirurgia estereotáxica. 2010. $131 \mathrm{f}$. Dissertação (Mestrado) -Instituto de Radioproteção e Dosimetria, Rio de Janeiro.

BEHARA, J.; FERANCIC, C. V.; SCAFF, L. A. M. Radiocirurgia estereotáxica no tratamento de malformações arteriovenosas cerebrais. Arquivos de Neuro-Psiquiatria, São Paulo, v. 45, n. 4, p. 391-396, 1987.

BRANDT, R. A.; SALVAJOLI, J. V.; OLIVEIRA,V. C.; CARMIGNANI, M.; CRUZ, J. C.; LEAL, H. D.; FERRAZ, L. Radiocirurgia por acelerador nuclear. Arquivos de Neuro-Psiquiatria, São Paulo, v. 53, n. 1, p. 38-45, 1995

BRASIL. Instituto Nacional de Câncer (Brasil). ABC do câncer: abordagens básicas para o controle do câncer / Instituto Nacional de Câncer - Rio de Janeiro: INCA, 2011.

BRASIL. Instituto Nacional de Câncer (Brasil). Ações de enfermagem para o controle do câncer: uma proposta de integração ensino-serviço / Instituto Nacional do Câncer - Rio de Janeiro: INCA, 2008.

BRASIL. Instituto Nacional de Câncer (Brasil). Estimativa 2016: incidência de câncer no Brasil / Instituto Nacional de Câncer - Rio de Janeiro: INCA, 2015.

CANTERA, M. M. Radiocirurgia. Revista Neurociências, São Paulo, v. 13, n. 4, p. 190-195, 2005.

CASTRO, D. G.; SALVAJORI, J. V.; CANTERAS, M. M.; CECILIO, S. A. J. Radiocirurgia nos adenomas hipofisários. Arquivos Brasileiros de Endocrinologia \& Metabologia, São Paulo, v. 50, n. 6, p.996-1004, 2006.

DELGADO, J. F.; VIEIRA, A. M. M.; CRUZ, J. C.; RODRIGUES, L. N. Cálculo independente de dose para tratamentos de arco dinâmico com colimador micromultilâminas. Revista Radiologia Brasileira, São Paulo, v. 39, n. 5, p. 355-359, 2006.

ESCOLASTICO, A. R. R. C. Radiocirurgia para tratamento de MAVs encefálicas. 2011. 92 f. Dissertação (Mestrado) - Faculdade de Medicina da Universidade de Lisboa, Lisboa.

FARIA, S. L.; SOUHAMI, L.; BAHARY, J. P.; CARON, J. L.; VILLEMURE, J. G.; OLIVER, A.; CLARK, B.; PODGORSAK, E. B. Metástase cerebral. Arquivos de Neuro-Psiquiatria, São Paulo, v.53, n.3-B, p. 570-576, 1995. 
FERNANDEZ, G. S. Impacto da radioterapia na qualidade de vida dos doentes com metastização cerebral. 2012. 93f. Dissertação (Mestrado) - Faculdade de Medicina da Universidade de Lisboa, Lisboa.

FILHO, G. B. Bogliolo patologia geral.Rio de Janeiro: Guanabara Koogan, 2013.

GOMES, J. G. R.; SAlVAJORI, J. V.; GORGUlHO, A.; SAlleS, A. A. F. Tratamento das metástases cerebrais com radiocirurgia: quando e como indicar? Jornal Brasileiro de Neurocirurgia, Rio de Janeiro, v.25, n.3, p. 240-250, 2014.

JOAQUIM, A. F.; GHIZONI, E.; TEDESCHI, H.; PEREIRA, E. B.; GIACOMINI, L. A. Radiocirurgia estereotáxica para metástases de coluna vertebral: Revisão de literatura. Revista do Instituto Israelita de Ensino E Pesquisa Albert Einstein, São Paulo, v. 11, n. 2, p. 247-255, 2013.

JUSTINO, P. B.; VILLAR, R. C.; CARVALHO, H. A.; NADALIN, W.; WELTMAN, E.. Radiocirurgia e radioterapia estéreotáxica: princípios e aplicações. Arquivos Brasileiros De Neurocirurgia, São Paulo, v. 23, n. 4, p. 163-170, 2004.

KONDZIOLKA, D.; PATEL, A.; LUNSFORD, L.D.; KASSAM, A.; FLICKINGER, J. C. Stereotactic radiosurgery plus whole brain radiotherapy versus radiotherapy alone for patients whit multiple brain metástases. International Journal of Radiation Oncology Biology Physics, Arlington, v. 45, n. 2, p. 427-434, 1999.

KOOY, H. M.; HERK, M. V.; BARNES, P. D.; ALEXANDER, E. III; DUNBAR, S. F.; TARBELL, N. J.; MULKERN, R. V.; HOLUPKA, E. J.; LOEFFLER, J. Image fusion for stereotactic radiotherapy and radiosurgery treatment planning. International Journal of Radiation Oncology Biology Physics, Arlington, v. 28, n. 5, p. 1229-1234, 1994.

LIMA, B. C.; JUNIOR, L. C. L.; LOPRETO, C. A. R.; JUNIOR, P. R. B.; BASAGLIA, R. Modalidades da radioterapia: teleterapia, braquiterapia e radiocirurgia. Revista Conexão Eletrônica,Três Lagoas, v. 11, n. 1, p. 165-174, 2014.

LOPES, A. C.; MATHIS, M. E.; CANTERAS, M. M.; SALVAJOLI, J. V.; PORTO, J.A.l; MIGUEL, E. C. Atualização sobre o tratamento neurocirúrgico do transtorno obsessivo-compulsivo. Revista Brasileira De Psiquiatria, São Paulo, v.26, n. 1, p. 62-66, 2004.

LUÍS, L.F. Custo-efetividade da radiocirurgia estereotáxica no tratamento de metástases cerebrais: revisão sistemática. Saúde \& Tecnologia: Revista Científica, Lisboa, n. 16, p. 1623, 2016.

LUNDGREN, M. S. F. S. Dosimetria em radiocirurgia para tumores cerebrais. 2011. 93f. Tese (Doutorado) Universidade Federal de Pernambuco, Recife.

LUNDGREN, M. S. F. S.; KHOURY, H. J.; AZEVEDO, S. Doses extracranianas em pacientes submetidos a radiocirurgia estereotáxica para tumores cerebrais. Revista Radiologia Brasileira, São Paulo, v. 45, n. 2, p. 83-86, 2012.

MAIA, E.T. Mapeamento de competências de profissionais de radioterapia em hospitais do SUS. 2015. 130 f. Dissertação (Mestrado)- Escola Nacional de Saúde Pública, Rio de Janeiro.

MALDAUN, M. V. C. Radiocirurgia no tratamento das metástases cerebrais: Resposta terapêutica e complicações baseadas na localização da lesão. 2006.128 f. Tese (Doutorado) Faculdade de Medicina da Universidade de São Paulo, São Paulo. 
MASCARENHAS, F.; COSTA, M. S.; OTRIZ, M.; ALMEIDA, A.; CARVALHO, H.; FERREIRA, A.G.; CATTONI, M.B. A radiocirurgia estereotáxica em tumores benignos e malignos do sistema nervoso central. Acta Médica Portuguesa, Lisboa, v.18, n. 1, p. 45-60, 2005.

METHA, M.P.; ROZENTAL, J.M.; LEVIN, A.B.; MACKIE, T.R.; KUBSAD, S.S.; GEHRING, M.A.; KINSELLA, T.J. Defining the role of radiosurgery in the management of brain metastases. International Journal of Radiation Oncology Biology Physics, Arlington, v. 24, n. 4, p. 619-625, 1992.

PANULLO, S. C.; YAMA, C.; WERNICKE, A.G. Diagnostic techniques and surgical management of brain tumors. Stereotactic radiosurgery for brain tumors. Rijeka: InTech, 2011.

SANTOS, A. J.; FRANCO, C. M. R.; BORGES, L. R. R.; MALHEIROS, S. M. F.; GABBAI, A. A. Metástases cerebrais. Revista Neurociências, São Paulo, v.9, n.1, p. 20-26, 2001.

SANTOS, W. M. Contribuição ao planejamento e à dosimetria de feixes de fótons aplicados à radiocirurgia e à radioterapia estereotáxica. 2003. 142 f. Tese (Doutorado) - Universidade Federal de Pernambuco, Recife.

SOUZA, C. N.; MONTI, C. R. Dosimetria dos cones radiocirúrgicos Radionics de diâmetros de 5mm a 50mm para um feixe de 6MV de um acelerador linear Mevatron MD digital. Revista Radiologia Brasileira,São Paulo, v.34, n.2, p. 95-100, 2001.

SOUZA, E. C. Resultados do tratamento radiocirúrgico de doentes com malformações arteriovenosas encefálicas classificadas como graus 3A, 3B, 4 ou 5 previamente submetidos ou não a embolização. 2010. 117 f. Tese (Doutorado) - Faculdade de Medicina da Universidade de São Paulo, São Paulo.

STURM, V.; KOBER, B.; HOVER, K.H.; SCHLEGEL W.; BOESECKE R.; PASTYR O.; HARTMANN G.H., SCHABBERT S.; WINKJZL K.Z.; KUNZE, S. LORENZ, W. J. Stereotactic percutaneous single dose irradiation of brain metástases with a linear accelerator. International Journal of Radiation Oncology Biology Physics, Arlington, v. 13, n. 2, p. 279-282, 1987.

TEIXEIRA, F. C. S. Estudo e desenvolvimento de um modelo de análise de risco para radiocirurgia intracraniana. 2015. 293 f. Tese (Doutorado) - Universidade do Estado do Rio de Janeiro, Rio de Janeiro.

WELTMAN, E.; BRANDT, R. Algoritmos para tratamento de metástases cerebrais. Einstein: educação continuada em saúde, São Paulo, v. 6, n. 2, p. 89-91, 2006. 